\title{
A multisystem approach by bed-side ultrasound in patients with COVID-19 infection: a case series
}

\author{
Gabriele Cioni, Ginevra Bellandi, Stefano Bertolini, Giacomo Betti, Wanda Bialek, Angela Fedeli, \\ Marco Antonio Lumini, Roberto Michelotti, Carmelo Muscari, Francesca Pacini, Sabrina Pastacaldi, \\ Rossella Squillante, Francesco Tola, Antonio Vergara, Emiliano Votino, Mauro Zuccotti, Fabio Daviddi \\ Emergency Department, SS Cosma and Damiano Hospital, Pescia, USL Toscana Centro, Italy
}

\begin{abstract}
Recent epidemiological reports on Chinese population affected by novel coronavirus showed a wide spread of clinical and biochemical alterations, suggesting a relationship between progression of lung damage to acute respiratory distress syndrome and the systemic inflammatory response, triggering an irreversible multiple organ damage and disseminated intravascular coagulation. Bedside ultrasound assessment provides integrated information, describing a multisystemic and dynamic clinical scenario for every patient. Furthermore, this approach allows to concentrate multiple information in the hands of a single operator, also limiting the risk of exposure to infection for healthcare professionals. As per our experience, herewith reported, we described the characteristics of 10 patients with SARS-CoV-2 infection. Ultrasound findings were related to clinical information, blood test analysis, and results of instrumental tests, such as chest X-ray and chest computed tomography. According to our ultrasound data, COVID-19 appears to be a systemic pathology even in those cases of mild to moderate disease. By this multisystem ultrasound approach, we could immediately recognize patients with a diffuse organ involvement and a more severe clinical pattern; moreover, we can protect healthcare workers and limit infection within health facilities.
\end{abstract}

Correspondence: Gabriele Cioni, Emergency Department, SS Cosma and Damiano Hospital, via Cesare Battisti 2, 51017 Pescia (PT), Italy.

Tel.: +39.0572.460.681.

E-mail: gabrielec.83@gmail.com

Key words: COVID-19; ultrasound; pneumonia.

Acknowledgments: thanks to all the nurses and first aid healthcare professionals of the Emergency Department of SS Cosma and Damiano Hospital of Pescia, who contributed to the management of the patients involved in the present case report.

Contributions: GC contributed to the design of the study, data collection and ultrasound evaluation, as well as to manuscript preparation; FD is the Emergency Department Director and approved both study and manuscript. All authors contributed to patient management and approved manuscript.

Consent: patients gave their informed consent to participate in the study.

Ethics: practice described for every single patient is consistent with our standard clinical care and comply with the Declaration of Helsinki.

Conflict of interests: the authors declare no potential conflict of interests.

Received for publication: 11 April 2020.

Accepted for publication: 14 April 2020.

This work is licensed under a Creative Commons Attribution NonCommercial 4.0 License (CC BY-NC 4.0).

${ }^{\circ}$ Copyright: the Author(s), 2020

Licensee PAGEPress, Italy

Italian Journal of Medicine 2020; 14:106-111

doi:10.4081/itjm.2020.1288

\section{Introduction}

The main cause of admission to the emergency room for COVID-19 patients is the presence of respiratory failure. An extensive lung involvement is the principal cause of clinical aggravation and admission to Intensive Care Unit (ICU). ${ }^{1-3}$

Due to progressive clinical instability, and high contagiousness, patients with COVID-19 cannot be easily moved from one setting to another in hospital facilities. ${ }^{4}$ For this reason, ultrasound method represents a fundamental resource in all the phases of their clinical management.

Scientific evidence showed the high reliability and sensitivity of ultrasound assessment as point-of-care in the critically ill patient. ${ }^{5}$ In particular, point-of-care ultrasound was the gold standard for clinical management in ICU and Emergency Department (ED). ${ }^{6}$ Moreover, several reports described the great efficiency of ultrasound in hemodynamic assessment and monitoring after therapy. ${ }^{7}$

The role of ultrasound in pneumonia diagnosis is well known. ${ }^{8}$

Recent epidemiological reports on Chinese population affected by novel coronavirus showed a wide spread of clinical and biochemical alterations, suggesting a relationship between progression of lung damage to acute respiratory distress syndrome (ARDS) ${ }^{9}$ and the systemic inflammatory response, triggering an irreversible multiple organ damage and disseminated 
intravascular coagulation. ${ }^{10}$ In this regard, the role of an increased prothrombotic response in COVID-19 pneumopathy and the frequent finding of pulmonary thromboembolism in patients with severe clinical course, may not be considered as a random association, but aspects of the same disease. ${ }^{11}$

Bedside ultrasound assessment provides integrated information, describing a multisystemic and dynamic clinical scenario for every patient. Furthermore, this approach allows to concentrate multiple information in the hands of a single operator, also limiting the risk of exposure to infection for healthcare professionals. ${ }^{6,12}$

\section{Case Report}

The aim of our study was to describe ultrasound findings in COVID-19 patients, in relation to clinical severity and instrumental and biochemical parameters.

The study population consists of 10 consecutive patients, affected by COVID-19, belonging to the ED of the SS Cosma and Damiano Hospital in Pescia, Italy, after the onset of fever and dyspnea.

According to current protocols we performed biochemical exams (whole blood cells count, transaminases, D-dimer, creatinine, $\mathrm{C}$ reactive protein, procalcitonin, high-sensitivity troponin, brain natriuretic peptide), arterial blood gas analysis, in order to assess respiratory function and lactate levels, and bedside chest X-ray.

Bedside ultrasound evaluation comprised the assessment of lung, cardiac function, liver parenchyma, diameters, and collapsibility of the inferior vena cava; it was performed during medical examination in the emergency department, by the same expert operator, using a 'ESAOTE MyLab 75' ultrasound, with a 3.5$\mathrm{MHz}$ convex probe.

Lung ultrasound was performed according to a method described elsewhere. ${ }^{13-15}$ During each exam 8 scans were obtained, 4 for each hemithorax, evaluating the upper and lower part of anterolateral regions.

Cardiac ultrasound assessment was performed according to the traditional transthoracic method.

To assess inferior cava vein measurements, the probe was positioned at the level of the subxiphoid region, with the patient in supine position. To acquire standardized measurements, the inferior vena cava (IVC) diameter was measured $2 \mathrm{~cm}$ from the junction between the inferior vena cava and the right atrium, recording in M-mode every 10 seconds, and including 2-3 cycles respirators. The inspiratory and expiratory diameters of the IVC were evaluated by measuring the lumen of the vein within the same respiratory cycle, measuring from the internal wall to the opposite internal wall. The measurements were collected in M-mode. ${ }^{16,17}$ We defined the inferior vena cava as collapsible in re- lation to the cut-off for the collapsibility index equal to $40 \%$, according to the literature data. ${ }^{17}$

The liver ultrasound evaluation was dedicated to the study of different parenchymal features on suspicion of acute organ damage. We assessed liver echogenicity in comparison with the renal parenchyma, pattern of homogeneity and inhomogeneity, visibility of vascular structures, visibility of the diaphragm, possible presence of portal vein thrombosis diaphragm. ${ }^{18}$

Based on our experience, we described the characteristics of 10 patients with SARS-CoV-2 infection. Ultrasound findings were related to clinical information, blood test analysis, and results of instrumental tests, such as chest X-ray and chest computed tomography (CT).

ICU admission was required for five patients due to the progressive worsening of respiratory failure.

Clinical, biochemical and instrumental characteristics of the study group were reported in Table 1.

ICU patients already showed severe hypocapnic respiratory failure upon presentation at emergency room; significant alterations on biochemical exams were found, in comparison with non-ICU patients. Chest X-rays showed widespread pulmonary thickening and interstitial plot reinforcement in ICU patients, while in others only uncommon mild interstitial disease was found.

In patients with more severe clinical impairment, ultrasound assessment discovered marked signs of organ damage. Ultrasound findings were reported in Table 1.

An extensive pulmonary involvement consisted in diffuse and confluent B lines, irregular pleural line and thickness, subpleural consolidations. Similar findings were described elsewhere for H1N1 pneumonia. ${ }^{8} \mathrm{My}-$ ocardial alterations, including wall brightness end pericardial effusion, were reported.

We did not find signs of hypovolemia; upon the assessment of vena cava diameters and collapsibility, we found a central venous overload, frequently associated with altered cardiac kinetic and a slight enlargement of right chambers. We excluded pulmonary embolism by CT angiography.

Non-ICU patients showed a mild respiratory involvement and minor biochemical alterations, requiring ordinary hospitalization. Chest X-ray was unremarkable; sporadic thickened pleural and rare subpleural consolidations, as well as isolated ground glass areas were reported on CT scan. Ultrasound evaluation was able to recognize signs of initial lung involvement even in patients with mild disease, showing sporadic non-confluent B lines, uncommon subpleural consolidations, and sporadic pleural thickening. Similar findings were described elsewhere. ${ }^{19}$ Cardiac function and central venous pattern were unremarkable in non-ICU patients. 
Ultrasound assessment of liver parenchyma showed irregular non-homogeneous brightness. On Doppler examination, demodulation of portal flow was also described. These findings were reported in ICU patients, but mild hyperechoic appearance of liver in comparison with renal parenchyma was also described in others. Ultrasound findings were shown in Figures 1 to 5.

\section{Discussion}

Novel coronavirus infection has been associated with widespread clinical presentation, ranging from respiratory and gastrointestinal symptoms, neurological and cardiological signs, suggesting a diffuse pattern of organ damage. COVID-19 pneumonia is the most widely described condition; other diseases related to novel coronavirus infection are lacking in evidence in recently published reports. However, a detailed investigation of the patient with advanced COVID-19 is not always possible due to the instability of the clinical conditions and the high risk of contagion for the clinical staff.

Bedside lung ultrasound could increase the quality of clinical information in the hands of the emergency physician, also limiting the exposure to the biological risk for healthcare professionals. Several studies

Table 1. Characteristics of study population.

\begin{tabular}{|c|c|c|}
\hline & Non-ICU patient $(n=5)$ & ICU patient $(n=5)$ \\
\hline $\mathrm{PaO}_{2}, \mathrm{mmH}_{2} \mathrm{O}($ mean $\pm \mathrm{SD})$ & $69 \pm 5$ & $49 \pm 7$ \\
\hline $\mathrm{PaCO}_{2}, \mathrm{mmH}_{2} \mathrm{O}($ mean $\pm \mathrm{SD})$ & $35 \pm 3$ & $24 \pm 4$ \\
\hline D-dimer, mcg/ifEU (mean \pm SD) & $750 \pm 78$ & $1820 \pm 250$ \\
\hline $\mathrm{CRP}, \mathrm{mg} / \mathrm{dL}(\mathrm{mean} \pm \mathrm{SD})$ & $5.3 \pm 2.3$ & $20.6 \pm 6.8$ \\
\hline HS troponin, pg/mL (mean $\pm \mathrm{SD})$ & $32.7 \pm 9$ & $100 \pm 12$ \\
\hline $\mathrm{AST}, \mathrm{U} / \mathrm{L}($ mean $\pm \mathrm{SD})$ & $55 \pm 8$ & $160 \pm 15$ \\
\hline $\mathrm{ALT}, \mathrm{U} / \mathrm{L}(\mathrm{mean} \pm \mathrm{SD})$ & $80 \pm 15$ & $230 \pm 21$ \\
\hline $\mathrm{LDH}, \mathrm{U} / \mathrm{L}($ mean $\pm \mathrm{SD})$ & $312 \pm 32$ & $530 \pm 37$ \\
\hline $\mathrm{WBC}, 1000 / \mathrm{mm} \times 3($ mean $\pm \mathrm{SD})$ & $4.1 \pm 0.3$ & $3.5 \pm 0.4$ \\
\hline Lactate levels, $\mathrm{mmol} / \mathrm{L}($ mean $\pm \mathrm{SD})$ & $1.2 \pm 0.2$ & $3.1 \pm 0.4$ \\
\hline
\end{tabular}

\section{Lung ultrasound}

\begin{tabular}{lcc}
\hline B lines & $\begin{array}{c}\text { Regional asymmetrical distribution; } \\
\text { focal pattern, non- confluent lines }\end{array}$ & $\begin{array}{c}\text { Diffuse, non-homogeneous, asymmetrical distribution, } \\
\text { spared areas of normal parenchyma; } \\
\text { multifocal pattern, confluent lines }\end{array}$ \\
\hline Pleural thickening & Regional distribution & $\begin{array}{c}\text { Irregular thickened fragmented pleural line, } \\
\text { findings diffuse on different quadrants }\end{array}$ \\
\hline Consolidations & Uncommon & Anterior sub-pleural consolidations \\
\hline Pleural effusion & No & Uncommon
\end{tabular}

\section{Cardiac ultrasound}

Pericardial effusion No

Frequent, non-circumferential, non-hemodynamic

\begin{tabular}{llc}
\hline Kinetic & No alterations & \multicolumn{1}{c}{ Regional hypokinesis } \\
\hline Chambers & No alterations & Slight increase in the right sections \\
\hline Myocardium & No alterations & Diffuse echo-bright appearance of the myocardium \\
\hline IVC ultrasound & & \\
\hline Diameter, mm $($ mean \pm SD) & $17 \pm 3$ & $22 \pm 3$ \\
\hline Collapsibility index, $\%(m e a n \pm S D)$ & Normal, $>40 \%$ & Venous congestion, reduced collapsibility index, $<40 \%$ \\
\hline
\end{tabular}

\section{Liver ultrasound}

Parenchymal pattern

\section{Hyperechogenic areas with regional distribution, predominantly normal parenchyma}

Significant hepato-renal difference, hyperechogenic areas with diffuse distribution, inhomogeneous areas of the parenchyma with regional distribution

ICU, Intensive Care Unit; $\mathrm{SD}$, standard deviation; $\mathrm{PaO}_{2}$, partial arterial pressure of $\mathrm{O}_{2} ; \mathrm{PaCO}_{2}$, partial arterial pressure of $\mathrm{CO}_{2}$; $\mathrm{CRP}$, $\mathrm{C}$ reactive protein; $\mathrm{HS}$ troponin, high-sensitivity troponin; ALT, alanine aminotransferase; AST, aspartate aminotransferase; LDH, lactate dehydrogenase; WBC, white blood cells; IVC, inferior vena cava. 
showed the accuracy of lung ultrasound in detecting and monitoring lung diseases, such as pneumonia and ARDS. ${ }^{5,20}$ Furthermore, ultrasound is extremely sensitive in distinguishing initial alterations from widespread organ alteration and it could be used as a gravity screening, selecting candidate patients for second level diagnostic procedures. Our data confirm the results of the literature, suggesting that ultrasound is superior in comparison with radiography, in defining lung damage. In addition, recent studies suggest that the ability of chest $\mathrm{CT}$ to identify suspected cases for interstitial pneumonia is not superior to pulmonary ultrasound. ${ }^{19,20}$

In addition to respiratory tract involvement, myocardial damage has been reported. Epidemiological reports revealed elevated levels of myocyte necrosis markers, such as creatine kinase-MB, N-terminal probrain natriuretic peptide (NT-proBNP) and high-sensitivity troponin. Arrhythmias were described in $16.7 \%$ of the 138 hospitalized COVID-19 patients at
Zhongnan Hospital of Wuhan University. ${ }^{21}$ Moreover, macroscopic alterations of the myocardium and pericardium have been described by Liu et al. however, due to the limited clinical data, the certainty of the relationship between myocardial changes and COVID19 requires further investigation.

Our findings confirmed data reported in recent studies describing an involvement of the myo-pericardial tissue related to coronavirus infection, suggestive for a myopericarditis. ${ }^{22}$

The pathogenetic link between coronavirus infection and cardiovascular disease is widely debated. ${ }^{23}$

Authors described multiple mechanisms, including direct viral damage, hypoxemia for severe lung involvement, and systemic inflammatory reaction ${ }^{24}$ related to cytokine storm. ${ }^{25}$ The role of the ACE2 enzyme in the pathogenesis of cardiovascular complications has been suggested recently, in particular for the evidence relating to SARS..$^{25,26}$

The evaluation of the hemodynamic status in the

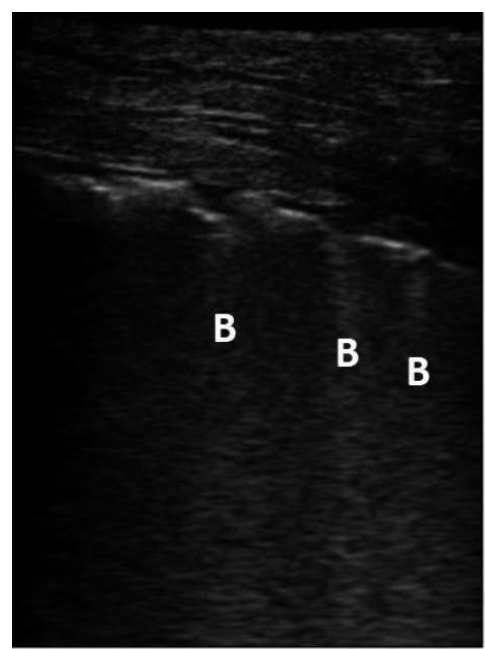

1

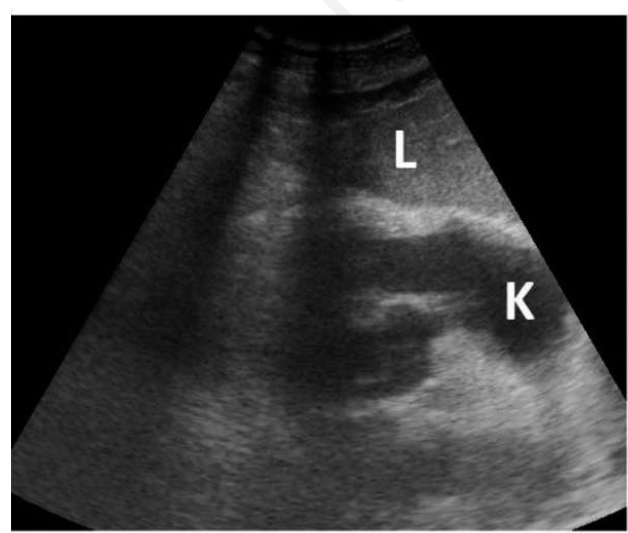

4

2
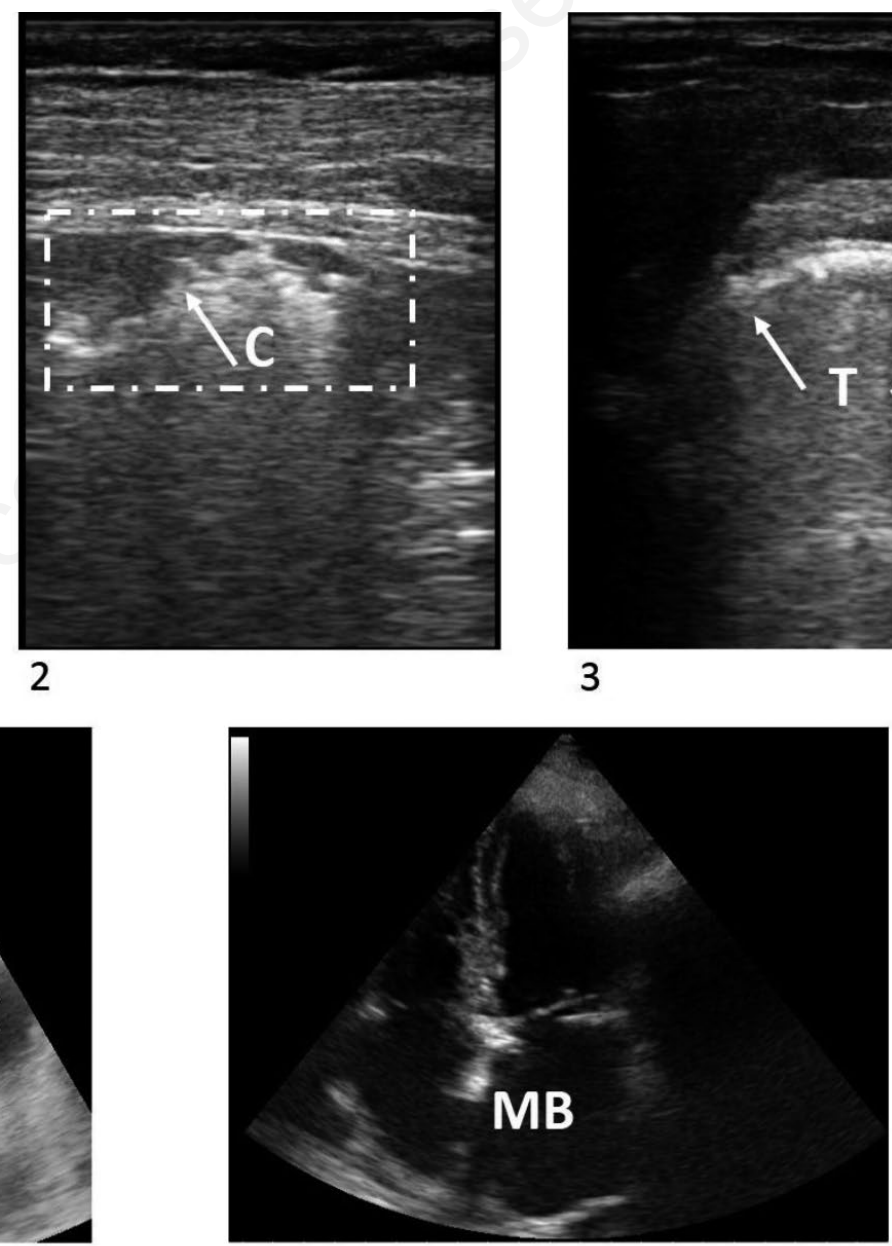

5

Figures 1-5. Ultrasound findings in COVID-19 patients, admitted to Intensive Care Unit. 1) B lines (B) and irregularities of the pleural line; 2) Sub-pleural consolidations (C); 3) Pleural thickening (T); 4) Inhomogeneous hyperechogenicity of the hepatic parenchyma $(\mathrm{L})$ in comparison with the renal parenchyma $(\mathrm{K})$; 5) Myocardial brightness (MB). 
critically ill patient is a key element of correct therapeutic management. Detailed reports on the hemodynamic status in patients with pneumonia due to COVID-19 are lacking. Several studies evaluated diagnostic utility of combined cardiac and thoracic critical care ultrasonography in optimizing differential diagnosis among the causes of respiratory failure, with particular reference to ARDS and pulmonary edema. Large IVC minimal diameter and diffuse $B$ lines were associated with cardiogenic edema, whereas a low B-line ratio was predictive of ARDS. ${ }^{27}$ In this report, we found a reduced collapsibility index and large diameter of IVC in patients with extensive pulmonary involvement; central venous congestion, not related to pulmonary edema, and a slight increase in the right sections could be associated with a diffuse pulmonary microvascular thrombotic damage, as suggested elsewhere.

Recent studies described gastrointestinal manifestations, including anorexia, nausea, vomiting and diarrhea; the possibility of fecal-oral transmission was suggested after detection of SARS-CoV-2 in the stool of infected patients. ${ }^{28}$ Varying degrees of abnormality in liver function indexes are frequently reported, and are apparently related to the systemic inflammatory state. ${ }^{29}$ Clinical implications of liver changes are not fully known, and there are no imaging investigations aimed at studying the liver parenchyma during coronavirus infection. In this case report, ICU patients showed ultrasound alteration of the hepatic parenchyma, which appeared hyperechoic, with an inhomogeneous structure. These structural changes are associated with alterations in liver function indexes. Further studies are needed to understand the role of these alterations at clinical level and to better understand the structural alterations of liver parenchyma.

\section{Conclusions}

According to our ultrasound data, COVID-19 appears to be a systemic pathology even in those cases of mild to moderate disease.

The multisystem ultrasound approach could provide essential information on the patient clinical condition and disease progression. For this purpose, the usefulness of chest radiography is limited, as the lesions are mainly evident in case of more advanced disease; chest CT, on the other hand, while providing a clear description of lung parenchyma, is not always executable, depending on the patient clinical condition, and does not provide dynamic indications. By this multisystem ultrasound approach, we could immediately recognize patients with a diffuse organ involvement and a more severe clinical pattern; moreover, we can protect healthcare workers and limit infection within health facilities.

\section{References}

1. Li L, Huang T, Wang YQ, et al. 2019 novel coronavirus patients' clinical characteristics, discharge rate and fatality rate of meta-analysis. J Med Virol 2020; Mar 12. [Epub ahead of print].

2. Xu XW, Wu XX, Jiang XG, et al. Clinical findings in a group of patients infected with the 2019 novel coronavirus (SARS-Cov-2) outside of Wuhan, China: retrospective case series. BMJ 2020;368:m606.

3. Wu C, Chen X, Cai Y, et al. Risk Factors associated with acute respiratory distress syndrome and death in patients with coronavirus disease 2019 pneumonia in Wuhan, China. JAMA Intern Med 2020; Mar 13. [Epub ahead of print].

4. Chen W, Huang Y. To protect healthcare workers better, to save more lives. Anesth Analg 2020; Mar 30. [Epub ahead of print].

5. Lichtenstein DA, Malbrain MLNG. Lung ultrasound in the critically ill (LUCI): a translational discipline. Anaesthesiol Intensive Ther 2017;49:430-6.

6. Buonsenso D, Piano A, Raffaelli F, et al. Point-of-care lung ultrasound findings in novel coronavirus disease19 pneumoniae: a case report and potential applications during COVID-19 outbreak. Eur Rev Med Pharmacol Sci 2020;24:2776-80.

7. Frankel HL, Kirkpatrick AW, Elbarbary M, et al. Guidelines for the appropriate use of bedside general and cardiac ultrasonography in the evaluation of critically ill patients-Part I: General ultrasonography. Crit Care Med 2015;43:2479.

8. Soldati G, Smargiassi A, Inchingolo R, et al. Is there a role for lung ultrasound during the COVID-19 pandemic? J Ultrasound Med 2020; Mar 20.[Epub ahead of print].

9. Tang X, Du R, Wang R, et al. Comparison of hospitalized patients with acute respiratory distress syndrome caused by COVID-19 and H1N1. Chest 2020; Mar 26. [Epub ahead of print].

10. Lillicrap D. Disseminated intravascular coagulation in patients with 2019-nCoV pneumonia. J Thromb Haemost 2020;18:786-7. [Epub 2020 Mar 24].

11. Danzi GB, Loffi M, Galeazzi G, Gherbesi E. Acute pulmonary embolism and COVID-19 pneumonia: a random association? Eur Heart J 2020; Mar 30. [Epub ahead of print].

12. Cheung JCH, Ho LT, Cheng JV, et al. Staff safety during emergency airway management for COVID-19 in Hong Kong. Lancet Respir Med 2020;8:e19.

13. Lichtenstein D. Fluid administration limited by lung sonography: the place of lung ultrasound in assessment of acute circulatory failure (the FALLS-protocol). Expert Rev Respir Med 2012;6:155.

14. Lichtenstein DA, Mezière GA, Lagoueyte JF, et al. Alines and B-lines: lung ultrasound as a bedside tool for predicting pulmonary artery occlusion pressure in the critically ill. Chest 2009; 136:1014.

15. Volpicelli G, Skurzak S, Boero E, et al. Lung ultrasound predicts well extravascular lung water but is of limited usefulness in the prediction of wedge pressure. Anesthesiology 2014;121:320.

16. Prekker ME, Scott NL, Hart D, et al. Point-of-care ultrasound to estimate central venous pressure: a compar- 
ison of three techniques. Crit Care Med 2013;41:833.

17. Nagdev AD, Merchant RC, Tirado-Gonzalez A, et al. Emergency department bedside ultrasonographic measurement of the caval index for noninvasive determination of low central venous pressure. Ann Emerg Med 2010;55:290.

18. Cioni G, Marcucci R, Abbate R, Boddi M. Semi-quantitative ultrasound assessment of nonalcoholic fatty liver disease highlightens early subclinical atherosclerotic vascular damage: from risk factors to vascular damage. Pol Ann Med 2020;27:13-20.

19. Peng QY, Wang XT, Zhang LN; Chinese Critical Care Ultrasound Study Group (CCUSG). Findings of lung ultrasonography of novel coronavirus pneumonia during the 2019-2020 epidemic. Intensive Care Med 2020; Mar 12. [Epub ahead of print].

20. Copetti R. Is lung ultrasound the stethoscope of the new millennium? Definitely yes! Acta Med Acad 2016; 45:80-1.

21. Wang D, Hu B, Hu C, et al. Clinical characteristics of 138 hospitalized patients with 2019 novel coronavirusinfected pneumonia in Wuhan, China. JAMA 2020;e201585:E1-E9. [Epub ahead of print].

22. Inciardi RM, Lupi L, Zaccone G, et al. Cardiac involvement in a patient with coronavirus disease 2019 (COVID-19). JAMA Cardiol 2020; Mar 27. [Epub ahead of print].

23. Guo J, Huang Z, Lin L, Lv J. Coronavirus disease 2019
(COVID-19) and cardiovascular disease: a viewpoint on the potential influence of angiotensin-converting enzyme inhibitors/angiotensin receptor blockers on onset and severity of severe acute respiratory syndrome coronavirus 2 infection. J Am Heart Assoc 2020;9:e16219. [Epub 2020 Apr 1].

24. Tan W, Jamil Aboulhosn J. The cardiovascular burden of coronavirus disease 2019 (COVID-19) with a focus on congenital heart disease. Int J Cardiol 2020; Mar 28. [Epub ahead of print].

25. Cioni G. The role of angiotensin-converting-enzyme 2 in the age- and sex related poor prognosis of COVID19. A comment on recent findings on novel coronavirus infection by SARS-CoV-2. Pol Ann Med 2020;27:85-7.

26. Matthay MA, Aldrich JM, Gotts JE. Treatment for severe acute respiratory distress syndrome from COVID-19. Lancet Respir Med 2020; Mar 20. [Epub ahead of print].

27. Sekiguchi H, Schenck LA, Horie R, et al. Critical care ultrasonography differentiates ARDS, pulmonary edema, and other causes in the early course of acute hypoxemic respiratory failure. Chest 2015;148:912-918.

28. Lee IC, Huo TI, Huang YH. Gastrointestinal and liver manifestations in patients with COVID-19. J Chin Med Assoc 2020; Apr 1. [Epub ahead of print].

29. Zhang Y, Zheng L, Liu L, et al. Liver impairment in COVID-19 patients: a retrospective analysis of 115 cases from a single center in Wuhan city, China. Liver Int 2020; Apr 2. [Epub ahead of print]. 